\title{
Multifrequency frequency-domain spectrometer for tissue analysis
}

\author{
Spichtig, S ; Hornung, R ; Brown, D W ; Haensse, D ; Wolf, M
}

\begin{abstract}
In this paper we describe the modification and assessment of a standard multidistance frequencydomain near infrared spectroscopy (NIRS) instrument to perform multifrequency frequency-domain NIRS measurements. The first aim of these modifications was to develop an instrument that enables measurement of small volumes of tissue such as the cervix, which is too small to be measured using a multidistance approach. The second aim was to enhance the spectral resolution to be able to determine the absolute concentrations of oxy-, deoxy- and total hemoglobin, water, and lipids. The third aim was to determine the accuracy and error of measurement of this novel instrument in both in vitro and in vivo environments. The modifications include two frequency synthesizers with variable, freely adjustable frequency, broadband high-frequency amplifiers, the development of a novel avalanche photodiode (APD) detector and demodulation circuit, additional laser diodes with additional wavelengths, and a respective graphic user interface to analyze the measurements. To test the instrument and algorithm, phantoms with optical properties similar to those of biological tissue were measured and analyzed. The results show that the absorption coefficient can be determined with an error of $<10 \%$. The error of the scattering coefficient was $<31 \%$. Since the accuracy of the chromophore concentrations depends on the absorption coefficient and not on the scattering coefficient, the $<10 \%$ error is the clinically relevant parameter. In addition, the new APD had similar accuracy as the standard photomultiplier tubes. To determine the accuracy of chromophore concentration measurements we employed liquid Intralipid® phantoms that contained $99 \%$ water, $1 \%$ lipid, and an increasing concentration of hemoglobin in steps of $0.010 \mathrm{mM}$. Water concentration was measured with an accuracy of $6.5 \%$ and hemoglobin concentration with an error of $0.0024 \mathrm{mM}$ independent of the concentration. The measured lipid concentration was negative, which shows that the current setup is not suitable for measuring lipids. Measurements on the forearm confirmed reasonable values for water and hemoglobin concentrations, but again not for lipids. As an example of a future application, chromophore concentrations in the cervix were measured and comparable values to the forearm were found. In conclusion the modified instrument enables measurement of water concentration in addition to oxy- and deoxyhemoglobin concentrations with a single source-detector distance in small tissue samples. Future work will focus on resolving the lipid component.
\end{abstract}

DOI: https://doi.org/10.1063/1.3082024

Posted at the Zurich Open Repository and Archive, University of Zurich

ZORA URL: https://doi.org/10.5167/uzh-17583

Journal Article

Published Version

Originally published at:

Spichtig, S; Hornung, R; Brown, D W; Haensse, D; Wolf, M (2009). Multifrequency frequency-domain spectrometer for tissue analysis. Review of Scientific Instruments, 80(2):024301. 
DOI: https://doi.org/10.1063/1.3082024 


\title{
Multifrequency frequency-domain spectrometer for tissue analysis
}

\author{
Sonja Spichtig, ${ }^{1,3}$ René Hornung, ${ }^{2}$ Derek W. Brown, ${ }^{1}$ Daniel Haensse,,${ }^{1,3}$ and \\ Martin Wolf ${ }^{1,3}$ \\ ${ }^{1}$ Clinic of Neonatology, University Hospital Zurich, 8091 Zurich, Switzerland \\ ${ }^{2}$ Department of Obstetrics and Gynecology, Kantonsspital St. Gallen, 9007 St. Gallen, Switzerland \\ ${ }^{3}$ Institute for Biomedical Engineering, Swiss Federal Institute of Technology, 8092 Zurich, Switzerland
}

(Received 22 February 2008; accepted 1 January 2009; published online 23 February 2009)

In this paper we describe the modification and assessment of a standard multidistance frequency-domain near infrared spectroscopy (NIRS) instrument to perform multifrequency frequency-domain NIRS measurements. The first aim of these modifications was to develop an instrument that enables measurement of small volumes of tissue such as the cervix, which is too small to be measured using a multidistance approach. The second aim was to enhance the spectral resolution to be able to determine the absolute concentrations of oxy-, deoxy- and total hemoglobin, water, and lipids. The third aim was to determine the accuracy and error of measurement of this novel instrument in both in vitro and in vivo environments. The modifications include two frequency synthesizers with variable, freely adjustable frequency, broadband high-frequency amplifiers, the development of a novel avalanche photodiode (APD) detector and demodulation circuit, additional laser diodes with additional wavelengths, and a respective graphic user interface to analyze the measurements. To test the instrument and algorithm, phantoms with optical properties similar to those of biological tissue were measured and analyzed. The results show that the absorption coefficient can be determined with an error of $<10 \%$. The error of the scattering coefficient was $<31 \%$. Since the accuracy of the chromophore concentrations depends on the absorption coefficient and not on the scattering coefficient, the $<10 \%$ error is the clinically relevant parameter. In addition, the new APD had similar accuracy as the standard photomultiplier tubes. To determine the accuracy of chromophore concentration measurements we employed liquid Intralipid® phantoms that contained $99 \%$ water, $1 \%$ lipid, and an increasing concentration of hemoglobin in steps of $0.010 \mathrm{mM}$. Water concentration was measured with an accuracy of $6.5 \%$ and hemoglobin concentration with an error of $0.0024 \mathrm{mM}$ independent of the concentration. The measured lipid concentration was negative, which shows that the current setup is not suitable for measuring lipids. Measurements on the forearm confirmed reasonable values for water and hemoglobin concentrations, but again not for lipids. As an example of a future application, chromophore concentrations in the cervix were measured and comparable values to the forearm were found. In conclusion the modified instrument enables measurement of water concentration in addition to oxyand deoxyhemoglobin concentrations with a single source-detector distance in small tissue samples. Future work will focus on resolving the lipid component. () 2009 American Institute of Physics.

[DOI: $10.1063 / 1.3082024]$

\section{INTRODUCTION}

Biological tissue exhibits relatively low absorption in the near infrared range, $650-1000 \mathrm{~nm}$, and light in this wavelength range penetrates tissue relatively deeply. Near infrared spectroscopy (NIRS) has been successfully applied in medicine for a variety of noninvasive diagnostic purposes, e.g., to quantitatively investigate the oxygenation, blood flow, volume, or function of tissues such as the breast, muscle, or brain. ${ }^{1-4}$ Various techniques have been developed for this purpose. Continuous wave spectroscopy employs light sources with constant intensity to determine changes in attenuation. However, this enables only measurement of changes in chromophore concentrations and not absolute values. To determine absolute values the absorption $\left(\mu_{a}\right.$ in $1 / \mathrm{cm})$ and reduced scattering $\left(\mu_{s}^{\prime}\right.$ in $\left.1 / \mathrm{cm}\right)$ coefficients of tissue must be quantified. Chromophore concentrations can then be calculated from $\mu_{a}$ values. Measurement of $\mu_{a}$ values is achieved either by time-domain spectroscopy, which records the dispersion of extremely short pulses of light by tissue, or by frequency-domain spectroscopy, which assesses the phase shift $(\Theta)$ and amplitude $(A)$ of intensity modulated light. Here, we focus on frequency-domain spectroscopy. There are two basic frequency-domain modes: the multidistance mode, which measures $\Theta$ and $A$ at several different source-detector distances, but at a single modulation frequency, and the multifrequency mode, which measures $\Theta$ and $A$ at a single source-detector distance, but at several different modulation frequencies. ${ }^{5}$ The multidistance method has a higher signal to noise ratio but assumes a relatively large volume of homogeneous tissue of $\sim 250 \mathrm{ml}$. For many clinical applications this assumption is fulfilled. However, other clinical situations necessitate small volume measurements. To increase the scope of clinical applications and the 
potential of NIRS it is then desirable to enable measurements on smaller tissue volumes. The multifrequency method is ideally suited for smaller volumes of tissue in the range of $\sim 30 \mathrm{ml}$. This extends the range of clinical applications to smaller organs or tissues such as the cervix. While multidistance instruments are commercially available, ${ }^{5,6}$ multifrequency instruments are not yet available on the market.

Therefore the aims of this work are the following:

- Transform a commercial ISS Inc. (Champaign, Illinois, USA) frequency-domain multidistance instrument to a multifrequency mode instrument, which enables measurements on small tissues at a single source-detector distance,

- To enhance the spectral resolution to enable quantitative detection of oxy-, deoxy-, and total hemoglobin $\left(\mathrm{O}_{2} \mathrm{Hb}\right.$, $\mathrm{HHb}$, and $\mathrm{tHb}$ ), as well as water and lipid concentrations, and

- To test the accuracy and error of measurements, thus characterizing the properties of the novel instrument in vitro and in vivo.

\section{INSTRUMENTATION}

The commercially available ISS Inc. (Imagent $\left.{ }^{\circledR}\right)$ multidistance instrument that was transformed originally featured four photomultiplier tubes (PMTs) as detectors and 32 laser diodes as light sources, 16 at $690 \mathrm{~nm}$ and 16 at $830 \mathrm{~nm}$. The emitted light intensity is modulated at $110 \mathrm{MHz} .^{6}$ The light sources are time multiplexed, i.e., only one light source is on at a time. The light is coupled to fibers (400 $\mu \mathrm{m}$ diameter) that guide light to the tissue. After penetrating the tissue the light is received and guided back to the instrument by another fiber (3 mm diameter), where it is detected by a PMT modulated at $110005 \mathrm{kHz}$. The demodulated signal at $5 \mathrm{kHz}$ is amplified and digitized in a computer (Datel PCI416-M2 PCI ADC board). The computer also generates the timing signals (PCI-CTR05 PCI board). A software computes $\Theta$ and $A$ by fast Fourier transform (FFT) and calculates the $\mu_{a}$ and $\mu_{s}^{\prime}$ and absolute concentrations of $\mathrm{O}_{2} \mathrm{Hb}, \mathrm{HHb}$, $\mathrm{tHb}$, and the regional tissue oxygen saturation $\mathrm{StO}_{2}$. The sensor implements a multidistance geometry, i.e., usually $\Theta$ and $A$ are measured at four different distances ranging from 1.5 to $5 \mathrm{~cm}$. In theory, it is assumed that the tissue below the sensor is homogeneous. ${ }^{5}$ Thus, this technique has been successfully applied in many types of tissue with a volume of $>\sim 250 \mathrm{ml}$. It would however be advantageous to extend the range of applications to small volumes of tissue such as the cervix. Additionally, the commercial instrument detectors are insensitive to wavelengths above $880 \mathrm{~nm}$ and thus chromophores such as water and lipids, whose absorption peaks are above $900 \mathrm{~nm}$, cannot be detected.

\section{A. Modified ISS Inc. multifrequency frequency- domain instrument}

To extend the range of NIRS to smaller tissue volumes and to enable measurement of water and lipids, a commercially available Imagent $\AA$ has been modified in collaboration with ISS Inc.
To enable the multifrequency mode, the two original synthesizers with fixed frequencies were replaced by two frequency generators (PTS 500), whose frequency can be freely adjusted from 50 to $500 \mathrm{MHz}$. A new broadband amplifier delivers the modulation frequency to the laser diodes and detectors. The BOXY (ISS Inc.) software that controls the instrument was modified to control the two frequency generators. Compared to network analyzers ${ }^{7}$ our setup decreases the sample time from $\sim 60$ to $\sim 10$ s and enables continuous measurements at a time resolution better suited for physiological measurements.

To be able to also measure water and lipid, the instrument was modified to measure at more, and also at higher, wavelengths. This required modifications of both the light sources and detectors. In the multifrequency mode, only one laser diode is needed per wavelength. Thus, one laser board was equipped with eight different laser diodes at the following wavelengths (in brackets the chromophore/s for which the respective wavelength is relevant): $690 \mathrm{~nm}(\mathrm{HHb}), 692$ $\mathrm{nm}(\mathrm{HHb}), 764 \mathrm{~nm}(\mathrm{HHb}), 831 \mathrm{~nm}\left(\mathrm{O}_{2} \mathrm{Hb}\right), 872 \mathrm{~nm}\left(\mathrm{O}_{2} \mathrm{Hb}\right)$, $888 \mathrm{~nm}\left(\mathrm{O}_{2} \mathrm{Hb}\right.$, lipid), $914 \mathrm{~nm}$ (lipid, water), and $980 \mathrm{~nm}$ (water).

The standard PMT detectors (R926, Hamamatsu Photonics, Japan) of the ISS Inc. instrument have several disadvantages. They are relatively expensive, fragile (an excess of light may destroy the PMT, a situation that can easily occur in a clinical environment), and need a high supply voltage of $1 \mathrm{kV}$. Their spectral sensitivity decreases rapidly at $800 \mathrm{~nm}$, which makes it impossible to detect water and lipid spectra above $880 \mathrm{~nm}$. In addition the frequency response of the PMT decreases above $400 \mathrm{MHz}$. However, they have a high internal gain and a large detection area. For our low-level light application, we have chosen an avalanche photodiode (APD) as light detector because it offers a broader spectral range including the wavelengths required for water and lipid detection. The APD also has a higher sensitivity, improved quantum efficiency compared to the PMT detector, and a higher signal to noise ratio than a PIN photodiode. To implement the APD two circuit boards were designed and produced, one to generate the high voltage for the APD, and the APD demodulation board with the APD itself and all the filters.

The reverse bias voltage of the APD is delivered by the TR-0.2P high-voltage power supply (Matsusada). It has an output voltage of $0-200 \mathrm{~V}$ and temperature compensation. The voltage is controlled by a potentiometer.

The intensity of the laser diodes can be modulated from 50 to $500 \mathrm{MHz}$. The APD detector is modulated at a frequency that is always $5 \mathrm{kHz}$ above the laser diodes. Thus, at the detector the frequency is mixed down to $5 \mathrm{kHz}$. This is achieved by heterodyne modulation of the reverse voltage of the APD. It consists of high-voltage and high-frequency elements. These are connected by inserting a capacitor as a dc blocker on the high-frequency element and inductivity as a high frequency blocker on the high-voltage side on the APD demodulation board (Fig. 1).

For instance, when the incident light is modulated with $50 \mathrm{MHz}$ and the APD with $50 \mathrm{MHz}+5 \mathrm{kHz}$, then the resulting signal contains the $\Theta$ and $A$ information in a $5 \mathrm{kHz}$ wave. 


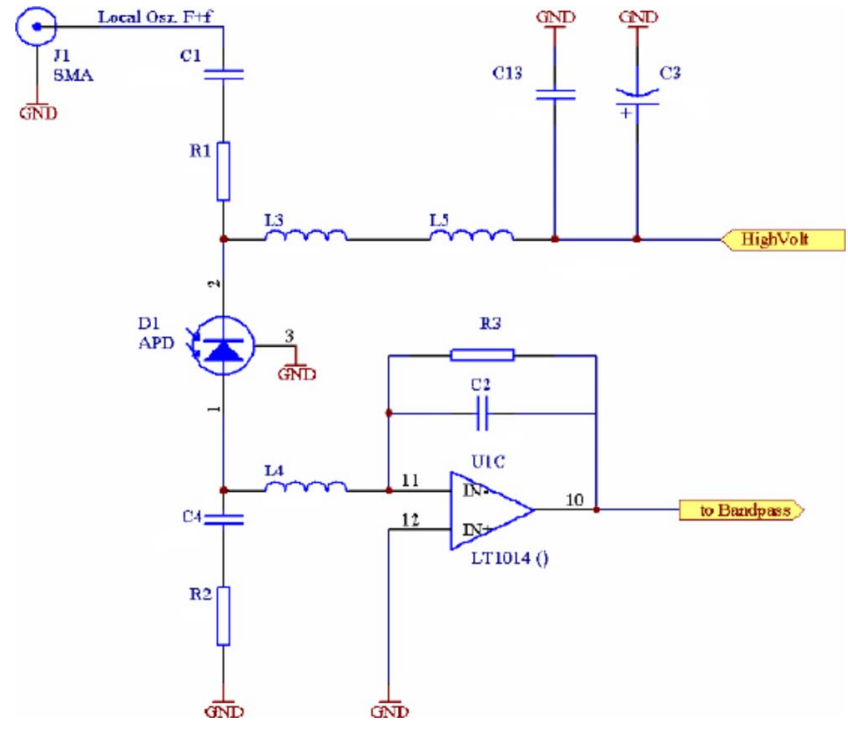

FIG. 1. (Color online) APD demodulation board, high voltage and high frequency parts of APD, lowpass and transimpedance amplifier.

The higher frequencies are suppressed by a lowpass filter, ${ }^{8}$ which is attached to the anode of the APD. It is followed by a transimpedance amplifier to convert the current into a voltage. A feedback capacitance over the operational amplifier is applied in order to avoid oscillations of the system. The 5 $\mathrm{kHz}$ signal is connected to an active bandpass filter, which is implemented as a two-pole (fourth order) multifeedback filter. ${ }^{9}$ It works with only one operational amplifier to serve the intended purpose. The source impedance must be low with respect to the input resistance with the transimpedance amplifier as the source. This implementation only allows a $Q$ value of 2 to 5 and the amplification is $-2 Q^{2}$. To ensure the stability of the system, the amplification of the operational amplifier should be at least $20 Q^{2}$ at the resonance frequency. An amplifier is connected to the output of the bandpass filter to enable reasonable values for the analog-digital converter board of the computer. The $\Theta$ and $A$ are calculated digitally by FFT.

\section{THEORY}

In the following we describe how the concentration of $\mathrm{O}_{2} \mathrm{Hb}, \mathrm{HHb}$, water, and lipid can be calculated from the $\Theta$ and $A$. The underlying theory of multifrequency frequencydomain spectroscopy has been described in detail elsewhere. $^{10}$ Briefly, the propagation of light through a highly scattering medium (absorption coefficient $\mu_{a}$ $\ll$ reduced scattering coefficient $\mu_{s}^{\prime}$ ) is described by the diffusion approximation to the Boltzmann transportation equation. The diffusion equation describes the propagation of a photon density wave

$$
-D(r) \nabla^{2} \Phi(r, t)+\mu_{a} \Phi(r, t)+\frac{1}{c} \frac{\partial \Phi(r, t)}{\partial t}=q_{0}(r, t)
$$

with $r$ as the source-detector distance, $t$ as the time, $q_{0}$ as the modulated point source, $D$ as the diffusion coefficient, and $\Phi(r, t)$ as the space- and time-dependent photon density. The light velocity in the medium can be calculated using $c=\left(3 \times 10^{8} \mathrm{~m} / \mathrm{s}\right) / n$, where $n$ is the refractive index for the medium.

The diffusion equation can be solved by assuming a semi-infinite medium, which approximately corresponds to the case in many clinical applications. The partial-currentextrapolated-boundary unification is an accurate representation of the boundary condition. For details about the theory and application see Ref. 11.

The two measurable quantities of a photon density wave, the phase lag $\Theta_{\text {lag }}^{m}$, and the amplitude $A_{\text {att }}^{m}$ can be theoretically simulated. For a given $\mu_{a}, \mu_{s}^{\prime}$, and modulation frequency $\omega$ of the point source, the solution to the diffusion equation can be solved and the theoretical phase lag $\Theta_{\text {lag }}^{\text {th }}\left(\omega, \mu_{a}, \mu_{s}^{\prime}\right)$ and amplitude attenuation $A_{\text {att }}^{\text {th }}\left(\omega, \mu_{a}, \mu_{s}^{\prime}\right)$ calculated. ${ }^{11}$

\section{A. Calibration and curve fitting}

Since the various instrument factors such as source power, detector gain, losses due to the source, and detector fiber are unknown and cannot be eliminated, the instrument needs to be calibrated. This calibration is done by measuring the phase lag $\Theta_{\text {lag }}^{m}$ and amplitude $A_{\text {att }}^{m}$ over all employed modulation frequencies on a phantom with known optical properties similar to tissue. A comparison between the obtained values with the theoretical phase lag $\Theta_{\text {lag }}^{\text {th }}\left(\omega, \mu_{a}, \mu_{s}^{\prime}\right)$ and amplitude attenuation $A_{\text {att }}^{\text {th }}\left(\omega, \mu_{a}, \mu_{s}^{\prime}\right)$ leads to the modulation-frequency-dependent correction factor $A_{\mathrm{att}}^{c}(\omega)$ and term $\Theta_{\text {lag }}^{c}(\omega)$,

$$
\begin{aligned}
& A_{\text {att }}^{c}(\omega)=\frac{A_{\text {att }}^{m}(\omega)}{A_{\text {att }_{\text {phantom }}^{\text {th }}}(\omega)}, \\
& \Theta_{\text {lag }}^{c}(\omega)=\Theta_{\text {lag }_{\text {phantom }}^{m}}^{m}(\omega)-\Theta_{\text {lag }_{\text {phantom }}^{\text {th }}}(\omega) .
\end{aligned}
$$

This correction factor and term are then applied to biological tissue measurements to eliminate the unknown instrumental factors

$$
A_{\mathrm{att}}^{m c}(\omega)=\frac{A_{\mathrm{att}}^{m}(\omega)}{A_{\mathrm{att}}^{c}(\omega)}, \quad \Theta_{\mathrm{att}}^{m c}(\omega)=\Theta_{\text {tissue }}^{m}(\omega)-\Theta_{\mathrm{lags} u}^{c}(\omega) .
$$

$A_{\text {att }}^{m c}(\omega)$ and $\Theta_{\text {lag }}^{m c}(\omega)$ are the calibrated measurement values of the tissue under investigation at a specific modulation frequency. A recursive fitting procedure is used to determine the $\mu_{a \text {,tissue }}$ and $\mu_{s \text {,tissue }}^{\prime}$ for which the corresponding $A_{\text {att }}^{\text {th }}(\omega)$ and $\Theta_{\text {lag }}^{\text {th' }}(\omega)$ show the best agreement to the calibrated measurement values $A_{\text {att }}^{m c}(\omega)$ and $\Theta_{\text {lag }}^{m c}(\omega)$. More explicitly, a least squares Gaussian-Newton algorithm with varying $\mu_{a \text {,fit }}$ and $\mu_{s, \mathrm{fit}}^{\prime}$ is applied to minimize the following expression over all modulation frequencies $\omega_{n}$ :

$$
\begin{aligned}
& \left\{\sum _ { n } \left(\left[A_{\mathrm{att}}^{\mathrm{th}}\left(\omega_{n}, \mu_{a, \mathrm{fit}}, \mu_{s, \mathrm{fit}}^{\prime}\right)-A_{\mathrm{att}}^{m c}\left(\omega_{n}, \mu_{a, \mathrm{tissue}}, \mu_{s, \mathrm{tissue}}^{\prime}\right)\right]^{2}\right.\right. \\
& \left.\left.+\alpha\left\{\Theta_{\text {lag }}^{\mathrm{th}}\left(\omega_{n}, \mu_{a, \mathrm{fit}}, \mu_{s, \mathrm{fit}}^{\prime}\right)-\Theta_{\text {lag }}^{m c}\left(\omega_{n}, \mu_{a, \mathrm{tissue}}, \mu_{s, \mathrm{tissue}}^{\prime}\right)\right\}^{2}\right)\right\} .
\end{aligned}
$$

The factor $\alpha$ adjusts the magnitude of the phase difference to that of the amplitude attenuation, meaning that differences in phase and amplitude have the same impact on the fitting 
procedure. It is also possible to fit $\Theta_{\text {lag }}$ or $A_{\text {att }}$ by themselves (minimizing the differences for each variable separately); however, fitting both simultaneously increases the robustness of the fit. ${ }^{12}$

\section{B. Determining the physiological properties}

Once $\mu_{a}$ and $\mu_{s}^{\prime}$ of the measured tissue have been determined, the physiological concentrations of interest, mainly $\mathrm{O}_{2} \mathrm{Hb}, \mathrm{HHb}$, water, and lipid, can be calculated. For a given wavelength $\lambda$, the relation between $\mu_{a}$ and the concentration of the mentioned absorbers is

$$
\begin{aligned}
\mu_{a}^{\lambda}= & \varepsilon_{(\mathrm{HHb})}^{\lambda} c_{(\mathrm{HHb})}+\varepsilon_{\left(\mathrm{O}_{2} \mathrm{Hb}\right)}^{\lambda} c_{\left(\mathrm{O}_{2} \mathrm{Hb}\right)}+\varepsilon_{\left(\mathrm{H}_{2} \mathrm{O}\right)}^{\lambda} c_{\left(\mathrm{H}_{2} \mathrm{O}\right)} \\
& +\varepsilon_{(\text {lipid })}^{\lambda} c_{(\text {lipid })},
\end{aligned}
$$

where $\varepsilon_{(x)}^{\lambda}$ is the molar extinction coefficient of absorber $x$ at wavelength $\lambda$ and $c_{(x)}$ is the concentration of absorber $x$. Equation (5) can also be written in the matrix form

$$
\vec{\mu}_{a}=\ln (10) \cdot E \cdot \vec{c},
$$

where $E$ is the extinction matrix and $c$ the concentration vector $^{13}$

$$
E=\left(\begin{array}{cccc}
\varepsilon_{\mathrm{HHb}, \lambda_{1}} & \varepsilon_{\mathrm{O}_{2} \mathrm{Hb}, \lambda_{1}} & \varepsilon_{\mathrm{H}_{2} \mathrm{O}, \lambda 1} & \varepsilon_{\text {lipid, }, \lambda_{1}} \\
\vdots & \vdots & \vdots & \vdots \\
\varepsilon_{\mathrm{HHb}, \lambda_{n}} & \varepsilon_{\mathrm{O}_{2} \mathrm{Hb}, \lambda_{n}} & \varepsilon_{\mathrm{H}_{2} \mathrm{O}, \lambda_{n}} & \varepsilon_{\text {lipid }, \lambda_{n}}
\end{array}\right), \quad \vec{c}=\left(\begin{array}{c}
c_{(\mathrm{HHb})} \\
c_{\left(\mathrm{O}_{2} \mathrm{Hb}\right)} \\
c_{\left(\mathrm{H}_{2} \mathrm{O}\right)} \\
c_{(\mathrm{lipid})}
\end{array}\right) .
$$

To calculate the concentrations in Eq. (6), the equation and hence the extinction matrix have to be inverted. In this case where the system is overdetermined (more wavelengths than absorbers), the Gaussian normal equation, a least-squares procedure minimizing the error, is applied,

$$
\vec{c}=\frac{1}{\ln (10)}\left(E^{T} \cdot E\right)^{-1} E^{T} \vec{\mu}_{a} .
$$

To calculate chromophore concentrations, the values of the extinction coefficients of hemoglobin and water were taken from the literature ${ }^{14-17}$ For lipid we used the spectra of pork lipid. ${ }^{16}$

\section{GRAPHICAL USER INTERFACE}

To analyze the data and calculate the optical properties a graphical user interface was programmed. It enables graphical inspection of the results of the calibration, measurement, and fitted lines. Furthermore, from the raw optical data the optical properties of the sample are calculated for each wavelength and the chromophore concentrations determined. Motion artifacts sometimes occur in clinical situations and may lead to unacceptable data. The graphic user interface enables immediate analysis and detection of such problems. Thus, often it will be possible to carry out another measurement without motion artifacts.

\section{MEASUREMENTS}

To test the reproducibility, reliability, and accuracy of the modified instrument and algorithms, several experiments were performed:

- Measurements on silicone phantoms with known optical properties,

- Measurements of an Intralipid® solution with known chromophore concentrations, and

- In vivo measurement on a male human forearm for a comparison with values from the literature. Additionally, measurements on the human cervix were performed to show the feasibility of the method for small tissue investigations.

For all measurements a single-distance reflection sensor with a source-detector separation of $1.5 \mathrm{~cm}$ was used (mean penetration depth of $\sim 5 \mathrm{~mm}$ ). The following eight laser diodes of the instrument were used: $980,914,888,872,805$, 764, 692, and $690 \mathrm{~nm}$. The measurements were recorded with modulation frequencies ranging from 130 to $490 \mathrm{MHz}$ in $10 \mathrm{MHz}$ steps. The modulation frequency range for the analysis of all measurements was constrained to $130-400$ MHz due to resonance effects below $130 \mathrm{MHz}$, significantly increasing noise for modulation frequencies above $400 \mathrm{MHz}$ and to optimize the signal to noise ratio.

\section{SILICONE PHANTOM MEASUREMENTS}

An array of four silicone phantoms with known optical coefficients (Table I) specified by the manufacturer ISS Inc. was selected to test the instrumental accuracy of the modified instrument. For comparison, the experiments were once performed with the APD and once with the PMT.

Each phantom was measured three times in an alternating order. First, the measurements were recorded by the PMT, then by the APD. For the analysis, a phantom (i.e., calibration phantom) was used to calibrate for the instrumental factors while optical properties of another phantom (i.e., measurement phantom) were measured. As the respective calibration phantom we chose the one with the $\mu_{a}$ closest to the one of the measurement phantom. The analysis was performed for each combination of a specific calibrationmeasurement phantom pair. For instance, for phantom 1 as measurement phantom and phantom 4 as calibration phantom, each measurement of phantom 1 was calibrated with each measurement of the calibration phantom 4. For three measurements on each phantom this yields nine combinations, i.e., nine estimates of $\mu_{a}$ and $\mu_{s}^{\prime}$ for the measurement phantom 1. $\mu_{a}$ and $\mu_{s}^{\prime}$ were averaged for each wavelength and the error $\left(100^{*}\right.$ (measured value-actual value)/actual value) and standard deviation (SD) of $\mu_{a}$ and $\mu_{s}^{\prime}$ calculated.

Since the spectral range of the PMT is limited to 880 $\mathrm{nm}$, the error for the wavelengths of 888,914 , and $980 \mathrm{~nm}$ were higher than $100 \%$ and increasing with the wavelength. For the other wavelengths, the error for $\mu_{a}$ was constant and $\leq 10 \%$. The APD showed no wavelength-dependent change in the accuracy of the measured optical properties, i.e., the error was very similar for all wavelengths for $\mu_{a}$ and $\mu_{s}^{\prime}$. For $\mu_{a}$ the error was $<10 \%$ for all wavelengths. Since the errors were very similar across wavelengths, in Table I the errors 
TABLE I. Results of the measurements on the silicone phantoms for the APD and the PMT detectors. The mean error and SD of the measured optical properties compared to the optical properties at $690 \mathrm{~nm}$ specified by the manufacturer ISS Inc. are listed.

\begin{tabular}{lcccccc}
\hline \hline \multicolumn{7}{c}{ Optical properties } \\
\cline { 3 - 5 } Detector & $\begin{array}{c}\text { Measurement } \\
\text { phantom no. }\end{array}$ & $\begin{array}{c}\mu_{a} \\
\left(\mathrm{~cm}^{-1}\right)\end{array}$ & $\begin{array}{c}\mu_{s}^{\prime} \\
\left(\mathrm{cm}^{-1}\right)\end{array}$ & $\begin{array}{c}\text { Calibration } \\
\text { phantom no. }\end{array}$ & $\begin{array}{c}\text { Error of } \mu_{a} \pm \text { SD } \\
(\%)\end{array}$ & $\begin{array}{c}\text { Error of } \mu_{s}^{\prime} \pm \text { SD } \\
(\%)\end{array}$ \\
\hline PMT & 1 & 0.125 & 4.9 & 4 & $4.77 \pm 2.15$ & $26.47 \pm 4.37$ \\
& 2 & 0.104 & 11.0 & 4 & $2.54 \pm 1.82$ & $0.63 \pm 0.33$ \\
& 3 & 0.146 & 4.1 & 1 & $10.00 \pm 1.18$ & $9.78 \pm 2.81$ \\
& 4 & 0.115 & 10.9 & 2 & $2.55 \pm 1.87$ & $0.63 \pm 0.39$ \\
APD & 1 & 0.125 & 4.9 & 4 & $3.70 \pm 2.07$ & $30.60 \pm 3.29$ \\
& 2 & 0.104 & 11.0 & 4 & $2.03 \pm 0.98$ & $1.63 \pm 0.61$ \\
& 3 & 0.146 & 4.1 & 1 & $9.90 \pm 2.77$ & $11.77 \pm 3.54$ \\
& 4 & 0.115 & 10.9 & 2 & $2.09 \pm 1.00$ & $1.65 \pm 0.57$ \\
\hline \hline
\end{tabular}

${ }^{\mathrm{a}}$ Measured at $690 \mathrm{~nm}$ by the manufacturer ISS Inc.

for $\mu_{a}$ and $\mu_{s}^{\prime}$ were averaged across all wavelengths for the APD and across all wavelengths $<880 \mathrm{~nm}$ for the PMT.

Table I shows that the error increases with the difference in the absorption between calibration and measurement phantom. For example the largest error was found for measurement phantom 3 and calibration phantom 1 with the largest $\Delta \mu_{a}=0.021 \mathrm{~cm}^{-1}$. The same is true for the $\mu_{s}^{\prime}$ : the errors for $\mu_{s}^{\prime}$ increase with the size of the $\Delta \mu_{s}^{\prime}$ between calibration and measurement phantom (e.g., for phantom 1 versus phantom $4 \Delta \mu_{s}^{\prime}=6 \mathrm{~cm}^{-1}$ and for phantom 2 versus phantom $\left.4 \Delta \mu_{s}^{\prime}=0.1 \mathrm{~cm}^{-1}\right)$. Since the accuracy of the chromophore concentrations depends on the $\mu_{a}$ and not on the $\mu_{s}^{\prime}$, the error in $\mu_{a}<10 \%$ is the clinically relevant parameter.

This has implications for measurements on tissue. It is advisable to use a calibration phantom with similar optical properties as the tissue under investigation. The observed effect may be due to some instrumental nonlinearities which lead to calibration errors that depend on the difference between calibration and measurement phantoms.

Summarizing the results, for all wavelengths the APD provides approximately the same accuracy as the PMT. However, the APD enables detection of wavelengths above $880 \mathrm{~nm}$, which includes absorption peaks of lipids and water. The small SD of the error demonstrates that the reproducibility of the measurements is high, demonstrating proper functioning of the instrument.

\section{INTRALIPID® MEASUREMENTS}

The main purpose of the measurements in an Intralipid ${ }^{\circledR}$ phantom was to test whether chromophore concentrations can be determined accurately. Thus, not only the instrumental accuracy but also the algorithms used to calculate optical properties and chromophore concentrations were tested. The Intralipid® phantom is a liquid emulsion, which has similar $\mu_{a}$ and $\mu_{s}^{\prime}$ to human tissue, and the concentrations of water, lipids, and $\mathrm{tHb}$ are known.

The APD detector, the same frequency range, and wavelengths were used as described above. Again, the calibration was performed with two silicone phantoms from ISS Inc. (phantoms 1 and 2 in Table I). The Intralipid ${ }^{\circledR}$ phantom consisted of $99 \%$ water with $1 \%$ Intralipid ${ }^{\circledR}$ and an increasing amount of hemoglobin. Intralipid( ${ }^{\circledR}$ without hemoglobin at this dilution is an emulsion of lipids, which has similar $\mu_{s}^{\prime}$ as tissue, i.e., $\mu_{s}^{\prime} \sim 9.71 \mathrm{~cm}^{-1}$ at $633 \mathrm{~nm} .{ }^{18}$ We added constant amounts of red cell concentrate [the hemoglobin content was determined beforehand by blood gas analysis (ABL 835, Radiometer Copenhagen)] in ten steps, increasing the $\mathrm{tHb}$ concentration by $0.010 \mathrm{mM}$ with each step. This $\mathrm{tHb}$ is used as the reference value and is called the "actual concentration of tHb." The Intralipid® solution was maintained at a constant temperature of $37{ }^{\circ} \mathrm{C}$ using a temperature controlled bath mixed with a magnetic mixer to ensure homogeneity of the solution and avoid sedation.

In total nine calibrations per silicone phantom were recorded: three before, three between, and three after the Intralipid@ phantom measurements. Each specific tHb concentration in the Intralipid ${ }^{\circledR}$ phantom was also measured three times and for the analysis every single measurement was calibrated with two (both phantoms) times nine calibration measurements. The resulting 54 optical coefficients for each $\mathrm{tHb}$ concentration of the Intralipid ${ }^{\circledR}$ phantom were then averaged to obtain mean and SD, which are displayed in Fig. 2.

With our instrument we determined the optical coefficients of $\mu_{a}=0.161 \mathrm{~cm}^{-1}$ and $\mu_{s}^{\prime}=11.56 \mathrm{~cm}^{-1}$ at $690 \mathrm{~nm}$. These coefficients were measured with $0.010 \mathrm{mM}$ of tHb. Since no data were recorded without hemoglobin in the In-

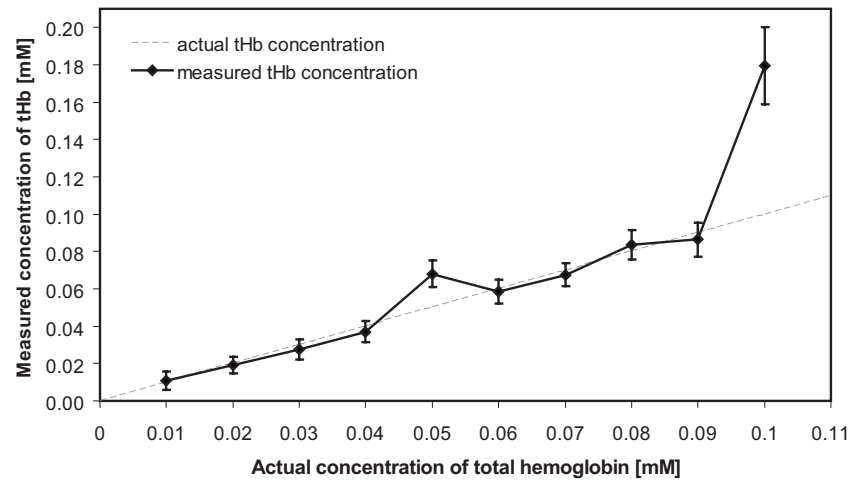

FIG. 2. Correlation of the actual tHb (dotted line) and the one measured by the modified NIRS instrument (solid line). 


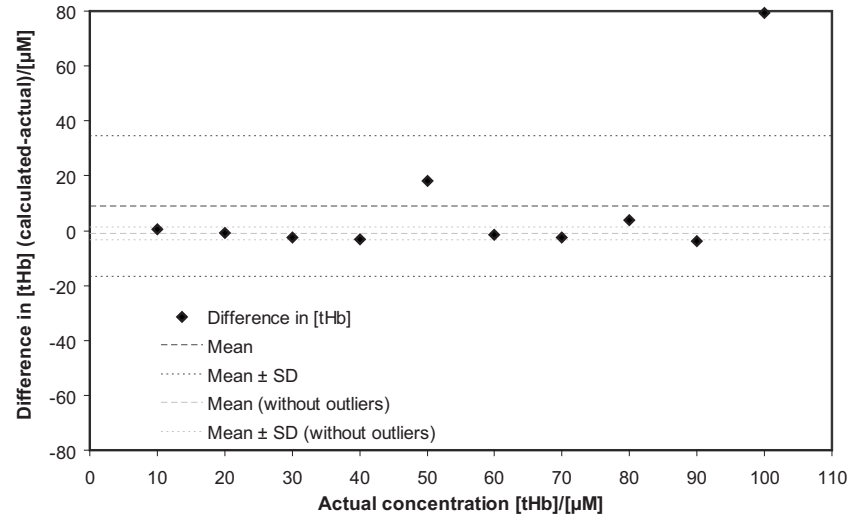

FIG. 3. Graphical representation of the difference in tHb concentration (measured-actual), the mean of the difference (prominent dotted line), \pm SD of the difference (dotted line) with and without outliers.

tralipid $\AA$ phantom, we subtracted the absorption of the $\mathrm{tHb}$ and received $\mu_{a}=0.049 \mathrm{~cm}^{-1}$ for the pure Intralipid@ phantom. Compared to the published optical coefficients ${ }^{18}$ of $\mu_{a}$ $\sim 0.0169 \mathrm{~cm}^{-1}$ and $\mu_{s}^{\prime} \sim 9.71 \mathrm{~cm}^{-1}$ at $633 \mathrm{~nm}$ the measured ones show good agreement, taking into account that the optical coefficients vary from batch to batch.

The measured $\mathrm{tHb}$ correlated well with the actual values (Fig. 2), except for the 0.050 and $0.100 \mathrm{mM}$ actual tHb. The reason for this error at 0.050 and $0.100 \mathrm{mM} \mathrm{tHb}$ is that the measured $\mu_{s}^{\prime}$ for the $980 \mathrm{~nm}$ wavelength were overestimated by a factor 3-4. This led to large errors in the water concentration but has also affected the tHb. Since our instrument is designed for tissue, which has a lower concentration of water than the Intralipid $®$ phantom with $99 \%$ water concentration, the signal at the $980 \mathrm{~nm}$ wavelength was relatively small because the absorption peak of water is at this wavelength. Therefore the signal to noise ratio at $980 \mathrm{~nm}$ was comparably low, which leads to high sensitivity to motion artifacts and probably led to the large $\mu_{s}^{\prime}$ values. In a real measurement in tissue, an error of this size would lead to the elimination and repetition of the measurement.

To analyze the agreement between the actual and measured $\mathrm{tHb}$ concentrations, we calculated the mean difference \pm standard deviation between the two concentrations (measured-actual) according to the literature: ${ }^{19}$ $\Delta[\mathrm{tHb}]=0.0088 \pm 0.02 \mathrm{mM}$ for all measurements (Fig. 3). These values are strongly affected by the two outliers 0.050 and $0.100 \mathrm{mM}$. When these were removed, a mean of the differences of $-0.0012 \pm 0.0024 \mathrm{mM}$ was obtained, which was 5-10 times smaller than the lowest measured concentration. This indicates a high degree of accuracy of the measured concentrations, provided outliers are detected by their erroneous $\mu_{s}^{\prime}$ and removed.

The concentration of water and lipid in the Intralipid ${ }^{\circledR}$ emulsion was nearly constant throughout the experimental series because the total volume of the solution was $400 \mathrm{ml}$ and the addition of $0.010 \mathrm{mM}$ of hemoglobin corresponded to an addition of only $1.6 \mathrm{ml}$ of red cell concentrate. Therefore, for water as well as lipid all measurements were averaged, resulting in a water concentration of $58.6 \pm 8.3 \mathrm{M}$. Since the Intralipid ${ }^{\circledR}$ phantom consisted of $99 \%$ water, a
TABLE II. Measured optical coefficients and chromophore concentrations for the forearm of a male subject.

\begin{tabular}{ccccc}
\hline \hline \multicolumn{5}{c}{ Human forearm } \\
\hline \multicolumn{5}{c}{ Optical properties } \\
$\mu_{a}$ \\
$(\mathrm{~nm})$ & $\begin{array}{c}\mu_{s}^{\prime} \\
\left(\mathrm{cm}^{-1}\right)\end{array}$ & Chromophore concentration \\
\hline 690 & $0.129 \pm 0.003$ & $8.10 \pm 0.91$ & {$[\mathrm{HHb}]$} & $0.0173 \pm 0.0006 \mathrm{mM}$ \\
692 & $0.124 \pm 0.007$ & $7.49 \pm 0.75$ & {$\left[\mathrm{O}_{2} \mathrm{Hb}\right]$} & $0.0421 \pm 0.005 \mathrm{mM}$ \\
764 & $0.141 \pm 0.006$ & $7.03 \pm 0.77$ & {$[\mathrm{tHb}]$} & $0.0594 \pm 0.005 \mathrm{mM}$ \\
831 & $0.130 \pm 0.007$ & $6.88 \pm 0.74$ & $\mathrm{StO}_{2}$ & $70.7 \pm 2.7 \%$ \\
872 & $0.164 \pm 0.014$ & $6.58 \pm 0.78$ & $\mathrm{H}_{2} \mathrm{O}$ & $22.98 \pm 1.34 \mathrm{M}$ \\
888 & $0.192 \pm 0.017$ & $6.46 \pm 0.71$ & $\mathrm{Lipid}$ & $-116.6 \pm 137.3 \mathrm{mM}$ \\
914 & $0.172 \pm 0.018$ & $5.9 \pm 0.63$ & & \\
980 & $0.330 \pm 0.007$ & $5.87 \pm 0.55$ & & \\
\hline \hline
\end{tabular}

concentration of $55 \mathrm{M}$ was expected. Thus, the water concentration was determined with a mean error of $6.5 \%$.

The lipid concentration of $-408 \pm 139 \mathrm{mM}$ was negative instead of close to $0 \mathrm{mM}$, which is erroneous. Thus, this experiment revealed that the lipid cannot be accurately measured with the current setup. There are two possible reasons for these erroneous results: (1) the implemented spectrum of porcine lipid may not match the actual spectrum of Intralipid $®$ and (2) the silicone of the phantom has an absorption peak at $910 \mathrm{~nm}$ close to the wavelength of $914 \mathrm{~nm}$. Although we have tried to correct for this absorption peak, the interpolation may not match the actual spectrum and we did not have the means to measure the actual spectrum of the calibration phantoms. We simulated small variations in the calibration of this peak and they considerably affected the measured lipid concentrations. Therefore we will measure the spectrum of the calibration phantom in the future in an attempt to overcome this problem. Once the spectra are obtained, the measured data can still be reanalyzed, but for the moment we conclude that the measurement of the lipid concentration is inaccurate.

In summary, the experiment demonstrates the importance of testing the accuracy of the instrument and algorithm. It also shows the feasibility of reliably and accurately measuring the $\mathrm{tHb}$ and water concentration but not lipid concentration by our instrument.

\section{IN VIVO MEASUREMENTS}

To test the modified instrument and analysis with an in vivo application, measurements on a male human forearm were performed and the results were compared to values previously presented in the literature. The APD detector, the same frequency range, and wavelengths were used as described above. For the calibration, two silicone phantoms from ISS Inc. (phantoms 1 and 2 of Table I) were used.

The measurements were performed in the following order: forearm, phantom 1 , and phantom 2 . The procedure was repeated three times. Each forearm measurement was calibrated with each phantom measurement and the mean and SD for each wavelength and chromophore concentration were calculated (Table II).

The measured concentrations of $\mathrm{HHb}, \mathrm{O}_{2} \mathrm{Hb}$, tHb, and 
TABLE III. Measured chromophore concentrations for the cervix of two test subjects.

\begin{tabular}{|c|c|c|c|}
\hline \multicolumn{4}{|c|}{ Cervix: chromophore concentration } \\
\hline \multicolumn{2}{|c|}{ Test subject 1} & \multicolumn{2}{|c|}{ Test subject 2} \\
\hline$[\mathrm{HHb}]$ & $0.0220 \mathrm{mM}$ & {$[\mathrm{HHb}]$} & $0.0145 \mathrm{mM}$ \\
\hline$\left[\mathrm{O}_{2} \mathrm{Hb}\right]$ & $0.0734 \mathrm{mM}$ & {$\left[\mathrm{O}_{2} \mathrm{Hb}\right]$} & $0.060 \mathrm{mM}$ \\
\hline$[\mathrm{tHb}]$ & $0.0954 \mathrm{mM}$ & {$[\mathrm{tHb}]$} & $0.0745 \mathrm{mM}$ \\
\hline $\mathrm{StO}_{2}$ & $76.9 \%$ & $\mathrm{StO}_{2}$ & $80.5 \%$ \\
\hline $\mathrm{H}_{2} \mathrm{O}$ & $24.95 \mathrm{M}$ & $\mathrm{H}_{2} \mathrm{O}$ & $19.56 \mathrm{M}$ \\
\hline Lipid & $-730.61 \mathrm{mM}$ & Lipid & $-766.65 \mathrm{mM}$ \\
\hline
\end{tabular}

$\mathrm{StO}_{2}$ values are similar to published data. ${ }^{20}$ Also the SDs of the hemoglobin and water concentrations are small, corresponding to an error of measurement of $3.5 \%$ for $\mathrm{HHb}$, $11.9 \%$ for $\mathrm{O}_{2} \mathrm{Hb}, 8.4 \%$ for $\mathrm{tHb}$, and $3.8 \%$ for $\mathrm{StO}_{2}$. Since it is expected that the concentration of the chromophores is not completely stable in living tissue, the true error may even be smaller.

A water concentration of $23.0 \pm 1.3 \mathrm{M}$ was obtained, which corresponds to a tissue water content of $41.37 \pm 2.41 \%$. From the literature, water concentration of $78 \pm 4 \%$ in the male human forearm muscle and $20 \pm 9 \%$ in the adipose tissue have been published. ${ }^{14}$ Since adipose tissue is present between muscle and the skin and due to the small mean penetration depth $(0.5 \mathrm{~cm})$ of the sensor, it is likely that mixture of muscle and adipose tissue was measured. A tissue water content of $41.37 \%$, i.e., a value between adipose tissue and muscle, seems reasonable. It is quite clear, however, that this is only a relatively imprecise estimate. The $\mathrm{SD}$ of repeated measurements and thus the error of measurement at $5.8 \%$ were small for water.

As in the Intralipid® experiment, the estimation of the actual lipid concentration yielded a negative value and thus was inaccurate. Possible reasons have been discussed above.

Thus, we conclude that for the $\mathrm{HHb}, \mathrm{O}_{2} \mathrm{Hb}, \mathrm{tHb}, \mathrm{StO}_{2}$, and water, the in vivo measurement yields reasonable values and an acceptable error of measurement for clinical research applications.

To show that the method is suitable for investigations of small tissue volumes, the same setup was used to perform measurements of the cervix on two test subjects. The results are shown in Table III. Since no other data on cervix chromophore concentrations have previously been published, we can only demonstrate the feasibility but not the accuracy of the method.

The measured $\mathrm{HHb}, \mathrm{O}_{2} \mathrm{Hb}$, and $\mathrm{tHb}$ are higher than the values on the male human forearm but still within a reasonable range. Water concentrations also compare favorably to those measured in the male human forearm. As before, estimation of the measured lipid concentration yielded a negative value and thus was inaccurate. Possible reasons for this have been discussed above.

In conclusion, in the cervix reasonable values for $\mathrm{O}_{2} \mathrm{Hb}$, $\mathrm{HHb}$, and water were obtained.

\section{CONCLUSIONS}

In this work we present the modification and assessment of a commercially available frequency-domain NIRS instrument adapted from a multidistance instrument to a multifrequency frequency-domain NIRS instrument to enable measurements in small volume of tissue. The algorithms used to determine optical coefficients and concentrations of $\mathrm{HHb}$, $\mathrm{O}_{2} \mathrm{Hb}, \mathrm{tHb}$, lipid, and water were implemented in a graphical user interface. The instrument was tested in solid and liquid phantoms as well as in in vivo settings. The results show that thorough testing in vitro and in vivo is essential to assess the strengths and weaknesses of an instrument and they demonstrate the capability of the modified instrument to accurately quantify both $\mu_{a}$ and $\mu_{s}^{\prime}$ and all chromophore concentrations (except for lipids) in highly scattering media, thus providing the necessary background information for further clinical investigations. There are numerous clinical applications for this noninvasive method, which include, but are not limited to, organs with small volumes such as the hand, foot, tongue, and cervix, the latter to monitor cervical ripening during pregnancy. ${ }^{21}$

\section{ACKNOWLEDGMENTS}

We gratefully acknowledge funding from the Swiss National Research Foundation.

${ }^{1}$ M. Wolf, G. Morren, D. Haensse, T. Karen, U. Wolf, J. C. Fauchere, and H. U. Bucher, Opto-Electron. Rev. 16, 413 (2008).

${ }^{2}$ D. R. Leff, O. J. Warren, L. C. Enfield, A. Gibson, T. Athanasiou, D. K. Patten, J. Hebden, G. Z. Yang, and A. Darzi, Breast Cancer Res. Treat. 108, 9 (2008).

${ }^{3}$ K. R. Ward, R. R. Ivatury, R. W. Barbee, J. Terner, R. Pittman, I. P. Filho, and B. Spiess, Resuscitation 68, 27 (2006).

${ }^{4}$ M. Wolf, M. Ferrari, and V. Quaresima, J. Biomed. Opt. 12, 062104 (2007).

${ }^{5}$ S. Fantini, M. A. Franceschini, J. B. Fishkin, B. Barbieri, and E. Gratton, Appl. Opt. 33, 5204 (1994).

${ }^{6}$ See http://www.iss.com/products/imagent/index.html for more information about the original version of the modified ISS instrument.

${ }^{7}$ T. H. Pham, O. Coquoz, J. B. Fishkin, E. Andersen, D. V. Gelfand, J. Milliken, T. Waddington, and B. J. Tromberg, Rev. Sci. Instrum. 71, 2500 (2000).

${ }^{8}$ S. Yokoyama, A. Okamoto, T. Araki, and N. Suzuki, Rev. Sci. Instrum. 66, 5331 (1995).

${ }^{9}$ D. Lancaster, Active Filter Cookbook (SAMS \& Inc, USA, 1981).

${ }^{10}$ S. R. Arridge, Inverse Probl. 15, R41 (1999).

${ }^{11}$ R. C. Haskell, L. O. Svaasand, T. T. Tsay, T. C. Feng, M. S. McAdams, and B. J. Tromberg, J. Opt. Soc. Am. A Opt. Image Sci. Vis 11, 2727 (1994).

${ }^{12}$ T. Pham, O. Coquoz, J. Fishkin, E. A. Anderson, and B. J. Tronberg, Rev. Sci. Instrum. 71, 2500 (2000).

${ }^{13}$ A. E. Cerussi, A. J. Berger, F. Bevilacqua, N. Shah, D. Jakubowski, J. Butler, R. F. Holcombe, and B. J. Tromberg, Acad. Radiol. 8, 211 (2001).

${ }^{14}$ S. J. Matcher, M. Cope, and D. T. Delpy, Phys. Med. Biol. 39, 177 (1994).

${ }^{15}$ S. J. Matcher, C. E. Elwell, C. E. Cooper, M. Cope, and D. T. Delpy, Anal. Biochem. 227, 54 (1995).

${ }^{16}$ Oregon Medical Laser Center, http://omlc.ogi.edu/spectra/.

${ }^{17}$ S. Wray, M. Cope, D. T. Delpy, J. S. Wyatt, and E. O. Reynolds, Biochim. Biophys. Acta 933, 184 (1988).

${ }^{18}$ H. J. van Staveren, C. J. M. Moes, J. van Marie, S. A. Prahl, and M. J. C. van Gemert, Appl. Opt. 30, 4507 (1991).

${ }^{19}$ J. M. Bland and D. G. Altman, Lancet 1, 307 (1986).

${ }^{20}$ C. Casavola, L. A. Paunescu, S. Fantini, and E. Gratton, J. Biomed. Opt. 5, 269 (2000).

${ }^{21}$ A. Banos, M. Wolf, C. Grawe, M. Stahel, D. Haensse, D. Fink, and R. Hornung, Lasers Surg. Med. 39, 641 (2007). 